\title{
Occurrence of Nanobacteria-Like Particles in Renal Stones of a Southern Brazilian Population
}

\author{
Amauri Braga Simonetti ${ }^{1}$, Carlos Teodósio Da Ros ${ }^{2 *}$, Cintia de David ${ }^{1}$, Karen Campos ${ }^{1}$, \\ Paulo Renato Petersen Behar ${ }^{3}$, Eduardo Ribeiro ${ }^{4}$, Gelsa Edith Navarro Hidalgo ${ }^{5}$ \\ ${ }^{1}$ Department of Microbiology, Federal University of Rio Grande do Sul, Porto Alegre, Brazil \\ ${ }^{2}$ Center of Andrology and Urology, Porto Alegre, Brazil \\ ${ }^{3}$ Department of Internal Medicine, Discipline of Infectious Diseases, \\ Universidade Federal de Ciências da Saúde de Porto Alegre (UFCSPA), Porto Alegre, Brazil \\ ${ }^{4}$ Ultralitho Medical Center, Florianópolis, Brazil \\ ${ }^{5}$ Department of Metallurgy, School of Engineering, Center of Technology, \\ Federal University of Rio Grande do Sul, Campus do Vale, Porto Alegre, Brazil \\ Email: *carlos.da.ros@terra.com.br
}

Received September 24, 2011; revised November 15, 2012; accepted December 3, 2011

\begin{abstract}
Purpose: Identifying the source of stone formation in recurrent stone formers has always been a big problem. Material and Methods: In this study kidney stones from 52 patients were submitted to direct examination by scanning electronic microscopy (SEM) after manual fracture and 27 calculi were cultured in Eagle's Minimum Essential Medium (E-MEM) and Brain-Heart Infusion (BHI) for eight weeks at $37^{\circ} \mathrm{C}$ in $5 \% \mathrm{CO}_{2}$ atmosphere. Twenty-seven powdered and demineralized stones were suspended in sterile PBS, filtered through $0.22 \mathrm{~m}$-pore-size sterile filters Millex (Millipore, Massachusetts, USA) and submitted to DNA extraction (Quiagen-Brazil). Platinum PCR SuperMIX (GIBCO-BRL), primers (Invitrogen), and Ultra Pure Water (Advanced Biotechnologies, Columbia, MD) were used for PCR (Polymerase Chain Reaction), which was generally conducted for 30 or 35 cycles with annealing of primers for $40 \mathrm{sec}$ at $55^{\circ} \mathrm{C}$ and extension for $1 \mathrm{~min}$ at $72^{\circ} \mathrm{C}$. Results: In 36 out $52(69 \%)$ kidney stones it was detected the presence of biofilm coating the mineral surface of the stone when examined by SEM, consisting of coccoid particles, isolated or clustered, with diameter of $500 \mathrm{~nm}$ or less. Eleven out 27 (41\%) kidney stone cultures produced white-colored sediment on the bottom of the tubes after eight-week incubation, revealing tiny structures similar to those observed directly by SEM. These structures were similar in size and morphology to spherical apatite particles previously observed in human kidney stones and named as nanobacteria (NB). No PCR products were observed in the samples. Conclusion: We found a strong correlation between renal stones and calcifying nanoparticles (CNP) in this study and these results open a new insight on this area to explore the etiology of stone formation. Whether NB/CNP are truly microorganisms or self-propagating mineral compounds is still controversial and its contribution, if any, in apatite nucleation and crystal growth remains uncertain.
\end{abstract}

Keywords: Nanobacteria; Calcifying Nanoparticles; Renal Stone

\section{Introduction}

The prevalence of nephrolithiasis has been increasing in the last years, reflecting the gradual rise in living standards, affecting up to $5 \%$ of the general population in the USA. While the exact etiology of this pathology is still not known, there have been many theories about stone pathogenesis taking into consideration several risk factors [1]. Stone formation in urinary tract is a multifactorial process that probably includes an imbalance between promoters of stone formation, such as urine supersaturation, crystal nucleation, aggregation and continued growth on the retained crystals, and their inhibitors, such as cit-

${ }^{*}$ Corresponding author. rate and urinary glycoproteins [2]. Epidemiological studies have shown that most people affected are idiopathic stone formers and that only up to $20 \%$ of patients with renal calculi have predisposing factors [3]. It is well established that urease-producing gram-negative organisms are involved in the formation of some renal stones, mainly Proteus, but this occurs in less than $10 \%$ of patients [4].

Nanobacteria (NB), also referred to as calcifying nanoparticles (CNP), have been described as structures raging from 80 to $500 \mathrm{~nm}$ in size, thus passing through $450 \mathrm{~nm}$ filters commonly used for media clarification, and even "sterilizing" filters (that is, $200 \mathrm{~nm}$ filters). NB are slow growing, not culturable in any standard microbiological 
medium, not Gram stainable, and resistant to heat and most antibiotics routinely used in cell culture. NB are calcified and self-propagating particles and can be observed either isolate as individual particles or in small groups or clumps [5]. Due to its capacity to produce apatite, it has been postulated that NB could be associated with extraskeletal calcification in a variety of pathological conditions [6]. Çiftçioglu et al. [7] found spherical apatite-formation in 14 renal samples, and in 13 of them, they identified CNP development.

Biomineralization in cell culture medium [8] yielded the formation of biofilms and mineral aggregates very similar to those found in tissue calcifications and renal pathologies, suggesting that NB could be efficient nuclei of mineralization, which start the formation of kidney stones [9]. Increasing number of reports has shown the role of NB in urologic diseases [10] and their occurrence in human renal pathologies such as polycystic kidney disease [5] and stone formations [11]. Researchers at NASA (National Aeronautics and Space Administration) have found that human NB multiply faster in a lowgravity environment, an observation deemed to be linked to the high risk of developing kidney stones for astronauts $[12,13]$. Kidney stone formation was reported to occur following injection of NB into rats $[14,15]$.

In spite of the possibility of NB being etiological agents of extracellular calcification there is still controversy on the nature of NB and their role on biomineralization. Major criticism is in relation to their small size, 10 to 100 times less than that of common bacteria, not sufficient for a living cell to contain all macromolecular components [16]. On the other hand, different authors question the existence of nucleic acids and NB capacity of biomineralization, pointing out that this process can be initiated by nonliving macromolecules and apatite itself [17].

The purpose of this study was to investigate the occurrence of biofilm and nanoparticles in kidney stones based on morphological evidence shown by scanning electron microscopy (SEM) and to propagate these particles in different cultural media. In this article NB/CNP is used to refer to nanobacteria.

\section{Material and Methods}

Kidney stones from 52 patients were included in this study. Informed consent was obtained from all patients. All stones were manually fractured with sterilized equipment and one of the fragments was used for direct examination by SEM.

Some stones, after powdered with a Potter device, were demineralized with $1 \mathrm{M} \mathrm{HCl}$ for $14-16$ hours, neutralized with $1 \mathrm{M} \mathrm{NaOH}$, centrifuged at $10,000 \times \mathrm{g}$ (Sigma $2 \mathrm{~K} 15$ ) for $10 \mathrm{~min}$ and the pellets washed 3 times with deionized water, each washing step intercalated by 10 min centrifugation. Stone fragments were deposited on glass coverslides, air dried and fixed with $1.25 \%$ glutaraldehyde (Merck) containing $4 \%$ sucrose in $0.5 \mathrm{M}$ phosphate buffer solution (PBS) for $16 \mathrm{~h}$ at $4^{\circ} \mathrm{C}$. Following extensive washing procedures with sterile PBS and rinsed in deionized water the fragments were dehydrated in a graded series of ethanol and treated with gold layer as described previously [8]. The samples were then examined by SEM (Philips E.M. 301).

Twenty seven powdered and demineralized stones were suspended in sterile PBS, filtered through $0.22 \mathrm{~m}-$ pore-size sterile filters Millex (Millipore, Massachusetts, USA) and inoculated into $5 \mathrm{ml}$ of BHI (Difco) and $5 \mathrm{~mL}$ of serum free E-MEM (Gibco) in $15 \mathrm{ml}$ tubes. All tubes were incubated at $37^{\circ} \mathrm{C}$ in a $5 \% \mathrm{CO}_{2}$ environment for eight weeks. Tubes were inspected macroscopically and microscopically for biofilm formation and calcification every week. Controls containing both media without stone material were treated the same way. These stones were submitted to DNA extraction (Quiagen-Brazil). Platinum PCR SuperMIX (GIBCO-BRL), primers (Invitrogen), and Ultra Pure Water (Advanced Biotechnologies, Columbia, MD) were used for PCR (Polymerase Chain Reaction), which was generally conducted for 30 or $35 \mathrm{cy}-$ cles with annealing of primers for $40 \mathrm{sec}$ at $55^{\circ} \mathrm{C}$ and extension for $1 \mathrm{~min}$ at $72^{\circ} \mathrm{C}$.

\section{Results}

In this study we demonstrated the presence of a layer resembling biofilm on the mineral surface of $69 \%$ of the stones analyzed by SEM, all showing coccoid nanoparticles bound together (Figure 1).

These particles were similar in size and morphology to spherical apatite particles previously observed in human kidney stones and named as nanobacteria [5,11,17]. After demineralization of these stones with $\mathrm{HCl}$ tiny coccoid structures, isolated or in clusters, were visualized by SEM (Figure 2). A remarkable occurrence was the variability of the particles size, but not exceeding $500 \mathrm{~nm}$, which is in agreement with that of $80-500 \mathrm{~nm}$ referred to NB [11].

Energy dispersive X-ray spectroscopy (EDS) indicated that the stones consisted mainly of calcium with the presence of phosphate and aluminum due to hydroxyapatite. The presence of carbon, even in low concentrations, reveals the existence of organic compound probably calcium oxalate (Figure 3) that is the main renal stone component according to the literature, with $80 \%$ of kidney stones containing calcium, and hypercalciuria occurring in $35 \%-40 \%$ of patients.

Eleven out 27 (41\%) kidney stone cultures were positive after eight-week incubation at $37^{\circ} \mathrm{C}$. With time the nanoparticles gradually increased in number turning both E-MEM and BHI media cloudy with a white-colored sedi- 


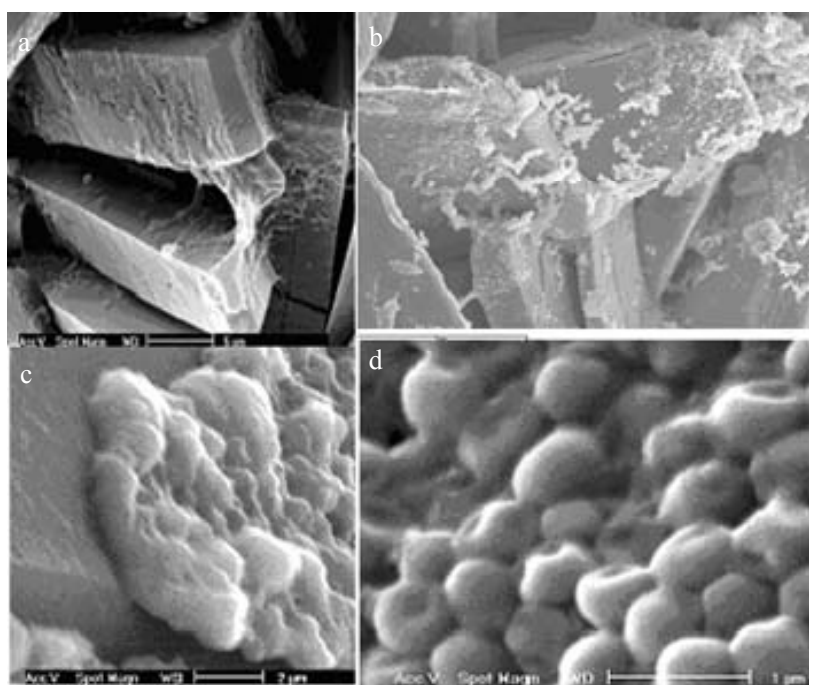

Figure 1. SEM micrograph of different kidney stones directly analyzed after fracturing. (a) A biofilm coating the stone mineral surface (bar $5 \mu \mathrm{m}, 3500 \times$ ); (b) Tiny clustered particles adhered to the crystal surface (bar $20 \mu \mathrm{m}, 3500 \times$ ); (c) Biofilm with particles grouped together (bar $2 \mu \mathrm{m}$, 8000×); (d) Coccoid structures showing rounded edge and division-like formations (bar $1 \mu \mathrm{m}, 25,000 \times)$.

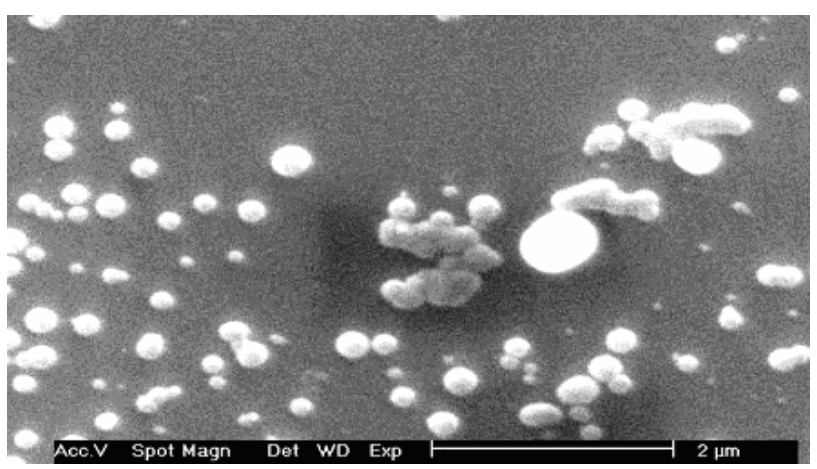

Figure 2. SEM micrograph of kidney stone after treatment with HCl. Coccoid structures lower than $500 \mathrm{~nm}$, isolated or in clusters, can be observed $(15,000 \times$; bar: $2 \mu \mathrm{m})$.

ment on the bottom of the tubes whereas the control did not show any growth (Figure 4). Analysis of this sediment by SEM showed nanoparticles of $500 \mathrm{~nm}$ or less, grouped in clusters (Figure 5). No PCR products were observed in the samples.

\section{Discussion}

Literature findings on the presence of NB/CNP in kidney stone material are controversial. While most authors found NB/CNP in kidney stones, Drancourt et al. [17] failed to isolate NB in cultured material from 10 samples, although detected nanoparticles in material analyzed by SEM. This discrepancy between results obtained by SEM direct analysis of renal stones and culture positivity is an intriguing one. In general, most reports have shown that analysis by SEM is more efficient than culture to detect $\mathrm{NB} / \mathrm{CNP}$. One question arises from this finding: if $\mathrm{NB} /$ $\mathrm{CNP}$ are precursors of renal stones, as claimed by many investigators, why culture is a less efficient detection method? One possibility is that for start growing NB/ CNP it would be necessary a minimum initial number of particles which would not occur in all stones. This might be related to the age of the stone. In this study we visualized a layer with nanoparticles in $69 \%$ of SEM analyzed stones but in only $41 \%$ of cultured stones nanoparticles were detected. If these structures are NB/CNP it means that in one third of the patients other factors or events would be responsible for stone formation. Kumon et al found ND/CNP in approximately $60 \%$ of the urinary stone samples between Japanese and Paraguayan patients [18].

It has been proposed that NB/CNP may act as a nucleus or nidi for mineral deposits and thus for kidney stone formation [11]. NB has also been detected in the kidney and urine after injection into rats [14,15]. However, other investigators contested the "nidi" hypothesis for NB/CNP suggesting that these structures are selfpropagating mineral compounds. The "nidi" hypothesis was again contested by Ryall's group [19], who demonstrated that $\mathrm{CaOx}$ monohydrate crystals precipitated spon-

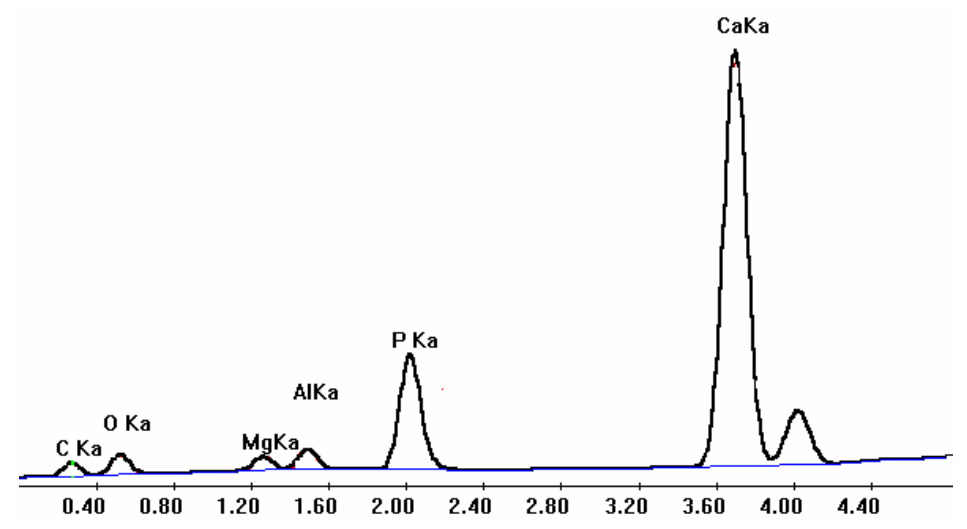

Figure 3. Composition of a human renal stone obtained by EDX. 


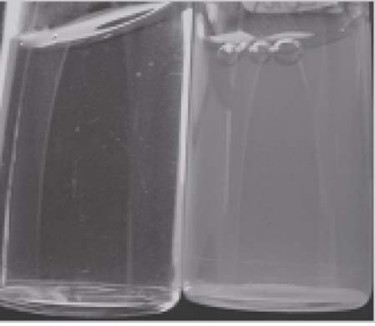

(a)

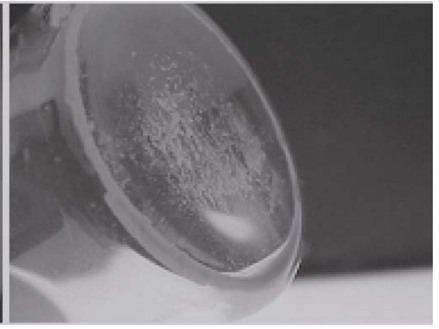

(b)
Figure 4. Images of material from a kidney stone cultured in BHI after eight-week incubation at $37^{\circ} \mathrm{C}$. (a) Tube at left side: control; tube at right side: kidney stone material; (b) White-colored sediment on the bottom of the tube.

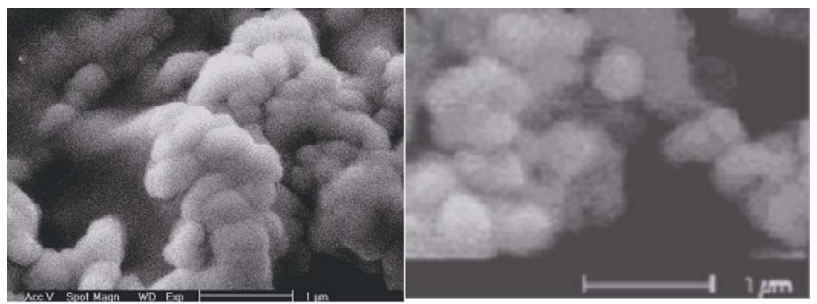

(a)

(b)

Figure 5. SEM micrographs of biofilm material from kidney stone cultures after eight-week incubation at $37^{\circ} \mathrm{C}$, showing tiny clustered particles in BHI (a) and E-MEM (b) (bar 1 $\mu \mathrm{m}, 20,000 \times$ ).

taneously from sterile human urine, suggesting that some, if not all, "nanobacteria" associated with pathological calcification might be nanoparticles formed spontaneously under conditions of high physiological supersaturation. Recently, Martel and Young [20] reported that calcium carbonate precipitates prepared in vitro were remarkably similar to purported NB in terms of their uniformly size, membrane-delineated vesicular shapes, with cellular division-like formations and aggregations in the form of colonies. These formations, consisting mainly of carbonate apatite, were also noticed by Kajander et al. [11] analyzing cultured material. We observed similar structures both in stones (Figures $\mathbf{1}$ and 2) and cultures (Figure 5). Interestingly, Folk [21] found structures named as nanobacteria in travertines, carbonate sediments and hot springs of central Italy, which are very similar to those described as NB/CNP. The author contends that they are responsible for a great deal of mineral precipitation as well as conversion of igneous mineral to soils, and the corrosion of metals.

\section{Conclusion}

We found a strong correlation between renal stones and CNP in this study and these results open a new insight on this area to explore the etiology of stone formation. Whether NB/CNP are truly nanoorganisms or self-propagating mineral compounds is still controversial and its con- tribution, if any, in apatite nucleation and crystal growth remains uncertain. Future studies are necessary for understanding the mechanisms involved in kidney stone formation.

\section{Acknowledgements}

The authors wish to thank to CNPq and CAPES for financial support and to Drs Túlio Graziottin and André Bonfanti for calculi supply.

\section{REFERENCES}

[1] I. P. Heilberg and N. Schor, "Renal Stone Disease: Causes, Evaluation and Medical Treatment," Brazilian Archives of Endocrinology and Metabolism, Vol. 50, No. 4, 2006, pp. 823-831. doi:10.1590/S0004-27302006000400027

[2] N. L. Miller, A. P. Evan and J. E. Lingeman, "Pathogenesis of Renal Calculi," Urologic Clinics of North America, Vol. 34, No. 3, 2007, pp. 295-313. doi:10.1016/j.ucl.2007.05.007

[3] M. Vella, M. Karydi, G. Coraci, R. Oriti and D. Melloni, "Pathophysiology and Clinical Aspects of Urinary Lithiasis," Urologia Internationalis, Vol. 79, Suppl. 1, 2007, pp. 26-31.

[4] M. J. Stechman, N. Y. Loh and R. V. Thakker, "Genetics of Hypercalciuric Nephrolithiasis: Renal Stone Disease," Annals of the New York Academy, Vol. 1116, No. 1, 2005, pp. 461-484.

[5] N. Ciftcioglu, D. S. McKay, G. Mathew and E. O. Kajander, "Nanobacteria: Fact or Fiction? Characteristics, Detection, and Medical Importance of Novel Self-Replicating, Calcifying Nanoparticles," Journal of Investigative Medicine, Vol. 54, No. 7, 2006, pp. 385-394. doi: $10.2310 / 6650.2006 .06018$

[6] E. O. Kajander, "Nanobacteria-Propagation Calcifying Nanoparticles," Letters in Applied Microbiology, Vol. 1242, No. 6, 2006, pp. 549-552.

[7] N. Ciftçioglu, K. Vejdani, O. Lee, G. Mathew, K. M. Aho, E. O. Kajander, D. S. McKay, J. A. Jones and M. L. Stoller, "Association between Randll's Plaque and Calcifying Nanoparticles," International Journal of Nanomedicine, Vol. 3, No. 1, 2008, pp. 105-115.

[8] A. B. Simonetti, G. E. Englert, K. Campos, M. Mergener, C. David, A. P. Oliveira, et al., "Nanobacteria-Like Particles: A Threat to Cell Cultures," Brazilian Journal of Microbiology, Vol. 38, No. 1, 2007, pp. 153-158.

[9] E. O. Kajander, N. Çiftçioglu, M. A. Miller-Hjelle and J. T. Hjelle, "Nanobacteria: Controversial Pathogens in Nephro- lithiasis and Polycystic Kidney Disease," Current Opinion in Nephrology and Hypertension, Vol. 10, No. 3, 2001, pp. 445-452. doi:10.1097/00041552-200105000-00023

[10] H. M. Wood and D. A. Shoskes, "The Role of Nanobacteria in Urologic Disease," World Journal of Urology, Vol. 24, No. 1, 2006, pp. 51-54.

[11] E. O. Kajander, N. Ciftcioglu, K. Aho and E. GarciaCuerpo, "Characteristics of Nanobacteria and Their Pos- 
sible Role in Stone Formation," Urological Research, Vol. 31, No. 2, 2003, pp. 47-54.

[12] N. Çiftçioglu, R. S. Haddad, D. C. Golden, D. R. Morrison and D. S. McKay, "A Potencial Cause for Kidney Stone Formation during Space Flights: Enhanced Growth of Nanobacteria in Microgravity," Kidney International, Vol. 67, No. 2, 2005, pp. 483-491.

[13] J. A. Jones, N. Cifticioglu, J. F. Schmid, Y. R. Barr and D. Griffith, "Calcifying Nanoparticles (Nanobacteria): An Additional Potential Factor for Urolithiasis in Space Flight Crews," Urology, Vol. 73, No. 1, 2009, p. 210.

[14] E. Garcia-Cuerpo, E. O. Kajander, N. Çiftçioglu, F. L. Castellano, C. Correa, J. Gonzálvez, et al., "Nanobacteria. An Experimental Neo-Lithogenesis Model," Archivos Españoles de Urología, Vol. 53, No. 4, 2000, pp. 291-303.

[15] F. A. Shiekh, M. Khullar and S. K. Singh, "Lithogenesis: Induction of Renal Calcifications by Nanobacteria," Urological Research, Vol. 34, No. 1, 2006, pp. 53-57. doi:10.1007/s00240-005-0034-0

[16] R. Psenner, M. Loferer and J. Maniloff, "Nannobacteria: Size Limits and Evidence," Science, Vol. 276, No. 5320, 1997, pp. 1773-1776.
[17] M. Drancourt, V. Jacomo, H. Lépidi, E. Lechevallier, V. Grisoni, C. Coulange, et al., "Attempted Isolation of Nanobacterium sp. Microorganisms from Upper Urinary Tract Stones," The Journal of Clinical Microbiology, Vol. 41, No. 1, 2003, pp. 368-372.

[18] H. Kumno, A. Matsumoto, S. Uehara, F. Abarzua, M. Araki, K. Tsutsui and K. Tomochika, "Detection and Isolation of Nanobacteria-Like Particles from Urinary Stones: Long-Withheld Data," International Journal of Urology, Vol. 18, No. 6, 2011, pp. 458-465.

[19] R. L. Ryall, “The Future of Stone Research: Rummagings in the Attic, Randall's Plaque, Nanobacteria, and Lessons from Phylogeny," Urological Research, Vol. 36, No. 2, 2008, pp. 77-97. doi:10.1007/s00240-007-0131-3

[20] J. Martel and J. D. Young, "Purported Nanobacteria in Human Blood as Calcium Carbonate Nanoparticles," Proceedings of National Academy of Science of the United States of America, Vol. 105, No. 14, 2008, pp. 5549-5554. doi:10.1073/pnas.0711744105

[21] R. L. Folk, "SEM Imaging of Bacteria and Nannobacteria in Carbonate Sediments and Rocks," Journal of Sedimentary Petrology, Vol. 63, No. 5, 1993, pp. 990-999. 